\title{
Differential Control of Glucocorticoid Receptor Hormone-Binding Function by Tetratricopeptide Repeat (TPR) Proteins and the Immunosuppressive Ligand FK506
}

\author{
Todd H. Davies, Yang-Min Ning, ${ }^{\ddagger}$ and Edwin R. Sánchez* \\ Department of Pharmacology, Medical College of Ohio, Toledo, Ohio 43614
}

Received July 14, 2004; Revised Manuscript Received October 16, 2004

\begin{abstract}
Many laboratories have documented the existence of tetratricopeptide repeat (TPR) proteins (also known as immunophilins) in hormone-free steroid receptor complexes. Yet, the distinct roles of these proteins in steroid receptor action are poorly understood. In this work, we have investigated the effects of four TPR proteins (FKBP52, FKBP51, Cyp40, and PP5) on hormone-binding function of glucocorticoid receptor (GR) endogenously expressed in mammalian L929 cells. As a first step, we treated L929 cells with select immunophilin ligands [FK506, rapamycin, cyclosporin A (CsA), and cyclosporin $\mathrm{H}(\mathrm{CsH})$ ], which are commonly thought to increase the GR response to hormone by inhibiting membranebased steroid exporters. As expected, all four immunophilin ligands increased both the intracellular concentration of dexamethasone and GR activity at the MMTV-CAT reporter. To determine whether these ligands could target GR function independent of steroid export mechanisms, we performed GR reporter gene assays under conditions of immunophilin ligand and dexamethasone treatment that yielded equal intracellular hormone concentrations. FK506 was found to stimulate GR transactivity beyond the effect of this ligand on hormone retention. In contrast, CsA only affected the GR through upregulation of hormone retention. By Scatchard analysis, FK506 was found to increase GR hormone-binding affinity while decreasing total binding sites for hormone. This result correlated with loss of GR-associated FKBP51 and replacement with PP5. Interestingly, no GR-associated Cyp40 was found in these cells, consistent with the ability of CsA ligand to only affect GR through the hormone export mechanism. To test the role of FKBP52 independent of FK506, FKBP52 was placed under the control of a tetracycline-inducible promoter. Upregulation of FKBP52 caused an increase in both GR hormone-binding affinity and transactivity, even in the absence of FK506. These results show that immunosuppressive ligands can alter GR hormone-binding function by changing the TPR protein composition of receptor complexes and that TPR proteins exert a hierarchical effect on this GR function in the following order: FKBP52 > PP5 > FKBP51.
\end{abstract}

The glucocorticoid receptor $(\mathrm{GR})^{1}$ is a ligand-activated transcription factor whose macromolecular structure in the absence of hormone contains heat shock protein 90 (Hsp90) and tetratricopeptide repeat (TPR) proteins. To date, four TPR proteins (FKBP52, FKBP51, Cyp40, and PP5) have been found in so-called "mature" GR complexes, i.e., GR that is competent for binding of hormone. Because Hsp90 contains only one binding site for these proteins, four distinct GR heterocomplexes have been identified on the basis of TPR protein content (reviewed in ref 1). Although such heterogeneity implies differential functions for each GR complex, very little is known about this process. In recent work by our laboratory, differential roles for FKBP52 and FKBP51 in the regulation of hormone-induced transport of GR were uncovered (2). It was found that binding of hormone caused GR complexes to shift from those that

\footnotetext{
This investigation was supported by a National Institutes of Health grant (DK43867) to E.R.S.

* To whom correspondence should be addressed. Phone: (419) 3834182. Fax: (419) 383-2871. E-mail: esanchez@mco.edu.

$¥$ Present address: Department of Internal Medicine, Wayne State University, 4201 St. Antonine, Suite 2E, Detroit, MI 48201-2022.
}

predominantly contain FKBP51 to complexes containing FKBP52. Simultaneous with this swapping event, recruitment of the motor protein dynein was observed, along with accumulation of the hormonally generated FKBP52-containing complex in the nucleus of cells. Thus, one role for GRassociated TPR proteins appears to be regulation of GR subcellular localization in the absence of hormone and transport of GR to the nucleus in its presence. Another role of GR-associated TPR proteins has been suggested by the works of Scammell, Smith, and others. In this case, the ability of GR to bind hormone appears to be regulated by the TPR ${ }^{1}$ protein content of the receptor complexes, with FKBP51 exhibiting an inhibitory role on this function and FKBP52 a stimulatory one (3-5).

FKBP52, FKBP51, and Cyp40 are receptors for the immunosuppressive drugs FK506 and cyclosporin A and,

\footnotetext{
${ }^{1}$ Abbreviations: GR, glucocorticoid receptor; TPR, tetratricopeptide repeat; FKBP, FK506-binding protein; Cyp40, cyclophilin 40; PP5, protein phosphatase 5; CsA, cyclosporin A; CsH, cyclosporin H; IP, immunophilin; Hsp90, heat shock protein 90; MMTV, mouse mammary tumor virus; CAT, chloramphenicol acetyl transferase; DMEM, Dulbecco's modified Eagle's medium.
} 
for this reason, are often referred to as immunophilins (6). Because FKBP52 present in GR heterocomplexes is capable of binding FK506 (7), it seems reasonable to expect that FK506 can target GR signaling events. Indeed, a variety of reports exist to support this notion. The first such report showed an ability of FK506 to potentiate GR-mediated reporter gene expression at low concentrations of hormone, while inhibiting gene expression at high concentrations (8). Similar results were observed for progesterone (9) and mineralocorticoid (10) receptors. FK506 modulation of GR activity at endogenous genes has also been found, such as $\alpha 1$-acid glycoprotein expression in the rat (11) and cyclooxygenase- 2 expression in human rheumatoid synovial fibroblasts (12). Although it is tempting to conclude that effects of FK506 on GR signaling occur by targeting FKBPs in GR heterocomplexes, a report by Yamamoto and coworkers suggests an unrelated mechanism in which FK506 causes inhibition of steroid hormone export from cells by targeting membrane-based P-glycoprotein exporters (13). Such a mechanism will result in higher intracellular concentrations of hormone following FK506 treatment of cells and would, thus, explain the ability of FK506 to potentiate GR actions at low concentrations of hormone. However, this mechanism cannot explain the reported ability of FK506 to repress GR-mediated gene activity at high hormone concentrations (8), nor does it explain the larger question of why FK506-binding immunophilins exist in GR heterocomplexes.

In this work, we have examined the mechanisms by which FK506 and TPR proteins lead to modulation of the GR hormone-binding and transcriptional enhancement activities. We show that FK506 can indeed increase the intracellular concentration of hormone. However, under conditions of equal intracellular hormone, FK506 continues to have a stimulatory effect on GR transactivity, which we show is due to loss of inhibitory FKBP51 from the GR complex followed by recruitment of PP5, leading to an increase in GR hormone-binding affinity. We also show that overexpression of FKBP52 leads to a dramatic increase in GR hormone-binding affinity, as well as increased GR reporter gene expression. Taken as a whole, these results serve to illustrate a complex interplay by which immunophilins, their cognate immunosuppressive ligands, and steroid exporters serve to modulate GR responses by targeting the earliest step in the steroid receptor signaling pathway, the hormonebinding function.

\section{EXPERIMENTAL PROCEDURES}

Materials. $\left[{ }^{3} \mathrm{H}\right]$ Acetate $(10.3 \mathrm{Ci} / \mathrm{mmol})$ was from $\mathrm{ICN}$ Radiochemicals (Costa Mesa, CA). $\left[{ }^{3} \mathrm{H}\right]$ Dexamethasone (43.2 $\mathrm{Ci} / \mathrm{mmol}$ ) was purchased from Dupont-New England Nuclear (Boston, MA). FK506 was from Fujisawa Pharmaceutical (Deerfield, IL). Rapamycin, cyclosporin A, and cyclosporin $\mathrm{H}$ were gifts from Dr. Jack-Michel Renoir of INSERM. All of the immunoligands were used at $10 \mu \mathrm{M}$ final concentration. Dexamethasone, methotrexate, G418 (geneticin) antibiotic, dextran, HEPES, DMEM-powdered medium, ironsupplemented calf serum, Hank's balanced salts, protease inhibitor cocktail, Tris, EDTA, sodium chloride, sodium acetate, coenzyme A, $S$-acetyl-coenzyme A synthetase, ATP, and chloramphenicol were obtained from Sigma (St. Louis, MO). The pMMTV-CAT reporter plasmid used for establishment of the four cell lines used in this study was from
Mark Danielsen and Gordon Ringold. It was constructed by placing the CAT reporter gene under the control of the complete MMTV long terminal repeat promoter where four consensus GRE elements are located. Transcriptional activity of the CAT gene is dependent on interaction of the activated GR with GREs (14). The pSV2neo plasmid was obtained from Margaret Hirst (Syntex). Under the control of SV40 promotor, this plasmid constitutively expresses aminoglycoside phosphotransferase, an enzyme responsible for resistance to neomycin and geneticin. The rabbit FKBP52 cDNA was also a gift from Dr. Renoir. It was placed into a tetinducible pBI-EGFP bidirectional expression vector from Clontech (Palo Atlo, CA).

Cell Lines and Culture. WCAT cells were established as previously described (15) by cotransfecting WCL2 cells with the pMMTV-CAT and pSV2neo plasmids, followed by selection with G418 (geneticin). The WCL2 cells (16) are derivatives of $\mathrm{CHO}$ cells that were subjected to rounds of methotrexate selection and amplification after cotransfection with the plasmids containing the cDNA for mouse wild-type GR and dihydrofolate reductase. WCL2 cells were maintained in $10 \mu \mathrm{M}$ methotrexate. The LMCAT cells were established by selection with G418 antibiotic following cotransfection with pMMTV-CAT and pSV2neo plasmids into mouse L929 fibroblast cells (8). To generate the L52MCAT cells used in this study, LMCAT cells were first stably transfected with the pUHD-172/Hygro plasmid, followed by hygromycin selection to yield LMCAT-Hygro cells. LMCAT-Hygro cells were then cotransfected with pBIFKBP52 and pCDNA6/His, a blasticidin selection plasmid. The resultant blasticidin-resistant L52MCAT cell line demonstrated high-level expression of rabbit FKBP52 after $48 \mathrm{~h}$ of doxycycline (D9891; Sigma) treatment. All cell lines were grown in an atmosphere of $5 \% \mathrm{CO}_{2}$ at $37{ }^{\circ} \mathrm{C}$ in DMEM and $10 \%$ iron-supplemented newborn calf serum. All serum used was prestripped of endogenous steroids by $1 \%$ (w/v) dextrancoated charcoal. All of the experiments were carried out on cells at or near confluence.

CAT Assay. CAT enzyme activity was measured by the method Nordeen et al. (17), with slight modification. Briefly, the cell lysates were made in $0.25 \mathrm{M}$ Tris and $3 \mathrm{mM}$ EDTA, $\mathrm{pH}$ 7.8, by three cycles of freezing and thawing and centrifugation at $13600 \mathrm{~g}$. Following a preincubation at 37 ${ }^{\circ} \mathrm{C}$ for $10 \mathrm{~min}$, the reaction was initiated by adding aliquots of lysates containing equal amounts of protein to the reaction mixture and incubating at $37^{\circ} \mathrm{C}$ for $2 \mathrm{~h}$. The reaction was stopped by addition of ice-cold benzene, which also served to extract the product $\left({ }^{3} \mathrm{H}\right.$-acetylated chloramphenicol) from the substrate $\left(\left[{ }^{3} \mathrm{H}\right]\right.$ acetate $)$ or intermediates $\left(\left[{ }^{3} \mathrm{H}\right]\right.$ acetyl-CoA $)$. The organic phases were dried in scintillation vials, and radioactivity was counted.

Intracellular Steroid Retention Assay. Replicate flasks of cells were pretreated for $30 \mathrm{~min}$ at $37{ }^{\circ} \mathrm{C}$ with the indicated immunosuppressants, followed by incubation with $\left[{ }^{3} \mathrm{H}\right]-$ dexamethasone at final concentrations of $10^{-9} \mathrm{M}$ (WCAT cells) or $10^{-8} \mathrm{M}$ (LMCAT cells) for an additional $4 \mathrm{~h}$. After thorough washing with $3 \times 10 \mathrm{~mL}$ volumes of cold Hank's balanced salt solution, the cells were suspended in $100 \mu \mathrm{L}$ volumes of hypotonic buffer $(10 \mathrm{mM}$ HEPES and $3 \mathrm{mM}$ EDTA, pH 7.4) and lysed by three cycles of freezing and thawing. To release $\left[{ }^{3} \mathrm{H}\right]$ dexamethasone-bound GR and other proteins from the nucleus, homogenates were extracted by 
addition of equal volumes of hypotonic buffer containing 1 $\mathrm{M} \mathrm{NaCl}$ ( $0.5 \mathrm{M}$ final concentration) and incubation on ice for $1 \mathrm{~h}$. Following centrifugation at $13600 \mathrm{~g}, 150 \mu \mathrm{L}$ aliquots of supernatant were counted, and the values were normalized to protein content.

Collection of PP5-Derived FLAG-TPR Peptide from Sf9 Cells. Isolation of the FLAG-TPR (a gift from Dr. Michael Chinkers and Dr. William Pratt) was derived from Chen et al. (18). FLAG-TPR-expressing baculovirus at a multiplicity of infection of 3 was used to infect a $100 \mathrm{~mL}$ suspension of Sf9 insect cells. Uninfected Sf9 cells were used as control. Infected and uninfected cells were incubated at $27^{\circ} \mathrm{C}$ for 3 days, then collected by centrifugation, and washed in $20 \mathrm{mM}$ HEPES and $150 \mathrm{mM} \mathrm{NaCl}, \mathrm{pH}$ 7.4. Collected pellets were suspended in $20 \mathrm{mM}$ HEPES and $50 \mathrm{mM} \mathrm{NaCl}, \mathrm{pH} 7.4$, plus protease inhibitor cocktail and sonicated for four $10 \mathrm{~s}$ intervals at output no. 5 on a Bronson Sonifier 250. The homogenate was centrifuged at $20000 \mathrm{~g}$ for $30 \mathrm{~min}$ at $4{ }^{\circ} \mathrm{C}$, and the supernatant was centrifuged at $125000 \mathrm{~g}$ for $30 \mathrm{~min}$ at $4{ }^{\circ} \mathrm{C}$. The final supernatant was tested for the presence of FLAG-TPR by Western blotting (data not shown). Samples were treated with $100 \mu \mathrm{g}$ of infected or uninfected Sf9 extract.

Cytosolic Steroid-Binding Assay. Replicate aliquots (45 $\mu \mathrm{L}$ ) of cytosol derived from LMCAT or L52MCAT cells, subjected to the indicated immunosuppressive ligand treatments (see figure legends), were incubated for $1 \mathrm{~h}$ at $4{ }^{\circ} \mathrm{C}$ with increasing concentrations of $\left[{ }^{3} \mathrm{H}\right]$ dexamethasone $(1-$ $100 \mathrm{nM}$ ) in the absence or presence of $50 \mu \mathrm{M}$ radioinert dexamethasone. This was followed by an additional incubation for $1 \mathrm{~h}$ at $37^{\circ} \mathrm{C}$. [It should be noted that effects of FK506 on hormone-binding function and GR heterocomplex composition (see below) did not occur without this warming step.] The free radiolabeled ligand was removed with $1 \%$ dextran-coated charcoal in 10 mM HEPES buffer ( $\mathrm{pH} 7.4$ ). Specific binding values were calculated and converted to femtomoles per milligram of cytosol protein. Calculation of $K_{\mathrm{d}}$ and $\beta_{\max }$ parameters was performed through computerized analysis (GraphPad Prism) using a one-site binding equation.

Immunoadsorption of GR Complexes. Mouse L929 cells were grown in DMEM containing 10\% charcoal-stripped calf serum (Hyclone Laboratories, Inc.). Cells were ruptured by Dounce homogenization in HEMG buffer (10 mM HEPES, $3 \mathrm{mM}$ EDTA, $20 \mathrm{mM}$ sodium molybdate, $5 \%$ glycerol, $\mathrm{pH}$ 7.4). Lysates were centrifuged at $20000 \mathrm{~g}$ for $30 \mathrm{~min}$. All cytosols were used without freezing or storage. In Figure 4, aliquots (typically $600 \mu \mathrm{L}$ ) of cytosol were treated for $1 \mathrm{~h}$ on ice with dexamethasone, FK506, appropriate vehicle controls, or combination treatments, followed by an additional incubation for $1 \mathrm{~h}$ at $37^{\circ} \mathrm{C}$. After rechilling, FiGR monoclonal ascites to GR [gift from Jack Bodwell (19)] or nonimmune mouse IgG2A was added for $2 \mathrm{~h}$. Samples were rotated with $20 \mu \mathrm{L}$ of protein $\mathrm{A}$-Sepharose at $4{ }^{\circ} \mathrm{C}$ overnight. The pellets were washed three times with TEG (10 mM Tris, $3 \mathrm{mM}$ EDTA, 10\% glycerol, $50 \mathrm{mM} \mathrm{NaCl}$, $20 \mathrm{mM}$ sodium molybdate, $\mathrm{pH}$ 7.4) followed by elution of GR complexes with $2 \times$ SDS.

Gel Electrophoresis and Western Blotting. Samples were resolved on denaturing SDS gels (20) using a 7-14\% acrylamide gradient in order to achieve maximal separation between the immunophilins and antibody heavy chains. Transfer of the samples to Immobilon-P membranes and quantitative immunoblotting were performed as previously described $(2,21,22)$. The FiGR monoclonal ascites against GR was used to probe for receptor, while various antibodies were used to probe for Hsp90 (H38220; Transduction Laboratories, Inc.), FKBP52 [UPJ56 (23)], FKBP51 (PA0021; Affinity Bioreagents), Cyp40 (PA3-022; Affinity Bioreagents), PP5 [a gift from Dr. Michael Chinkers (18)], and dynein intermediate chain (MAB 1618; Chemicon International, Inc.). The blots were then incubated with appropriate peroxidase- and ${ }^{125} \mathrm{I}$-conjugated counter antibodies, followed by color development and autoradiography.

\section{RESULTS}

FK506 Increases GR Transactivity Independent of Steroid Export Mechanisms. In previous work by our laboratory, we used mouse L929 cells stably transfected with the GRresponsive pMMTV-CAT reporter (LMCAT cells) to demonstrate a stimulatory effect by FK506 on GR transactivity $(8,24)$. Because these cells express P-glycoprotein exporter that is known to be sensitive to FK506 (13), we sought to test the ability of FK506 to potentiate GR in cells that were lacking this protein. Chinese hamster ovary $(\mathrm{CHO})$ cells (parental strain) are purported to be relatively free of $\mathrm{P}$-glycoprotein exporters, although multidrug-resistant $\mathrm{CHO}$ strains have been developed with elevated levels of $\mathrm{P}$ glycoprotein exporter (25). Initial tests of our $\mathrm{CHO}$ cells showed little effect by immunoligands on intracellular retention of the glucocorticoid agonist, dexamethasone (Dex). We therefore assessed the effect of FK506 in CHO cells that stably express wild-type mouse GR and the pMMTV-CAT reporter (WCAT cells) (15). In the experiments of Figure 1, WCAT and LMCAT cells were subjected to treatment with various immunosuppressive ligands, followed by assay for intracellular hormone content using $\left[{ }^{3} \mathrm{H}\right]$ dexamethasone, as well as assay for CAT reporter activity. LMCAT cells were exposed to $10^{-8} \mathrm{M}$ Dex, while WCAT cells received $10^{-9}$ M Dex. These conditions were chosen because these were the concentrations of Dex found to give minimal activity at the CAT reporter for each cell line (data not shown), an observation that reinforces the fact that $\mathrm{CHO}$ (WCAT) cells have a less active steroid export mechanism compared to L929 (LMCAT) cells. The results in LMCAT cells show that FK506, rapamycin, cyclosporin A (CsA), and cyclosporin $\mathrm{H}(\mathrm{CsH})$ all substantially increased intracellular hormone concentration and GR-mediated CAT activity. In WCAT cells, all four immunosuppressive ligands caused a potentiation of GR transactivity, yet the effect of these ligands on Dex retention was barely detectable, with the possible exception of FK506.

Although the above results suggest that immunoligands can directly target GR, we clearly needed a technique that would allow us to measure the effects of IP ligands on GR responses independent of steroid export mechanisms. To achieve this goal, we set out to determine whether immunoligands could still cause potentiation of GR under conditions of equal intracellular hormone concentration. These conditions were identified by measuring $\left[{ }^{3} \mathrm{H}\right]$ dexamethasone retention in LMCAT cells subjected to a constant amount of FK506 or CsA but varying amounts of hormone (data not shown). In Figure 2 (black bars), it can be seen that cells subjected to $3 \times 10^{-9}$ M hormone plus FK506 or CsA had the same intracellular hormone concentration as cells sub- 

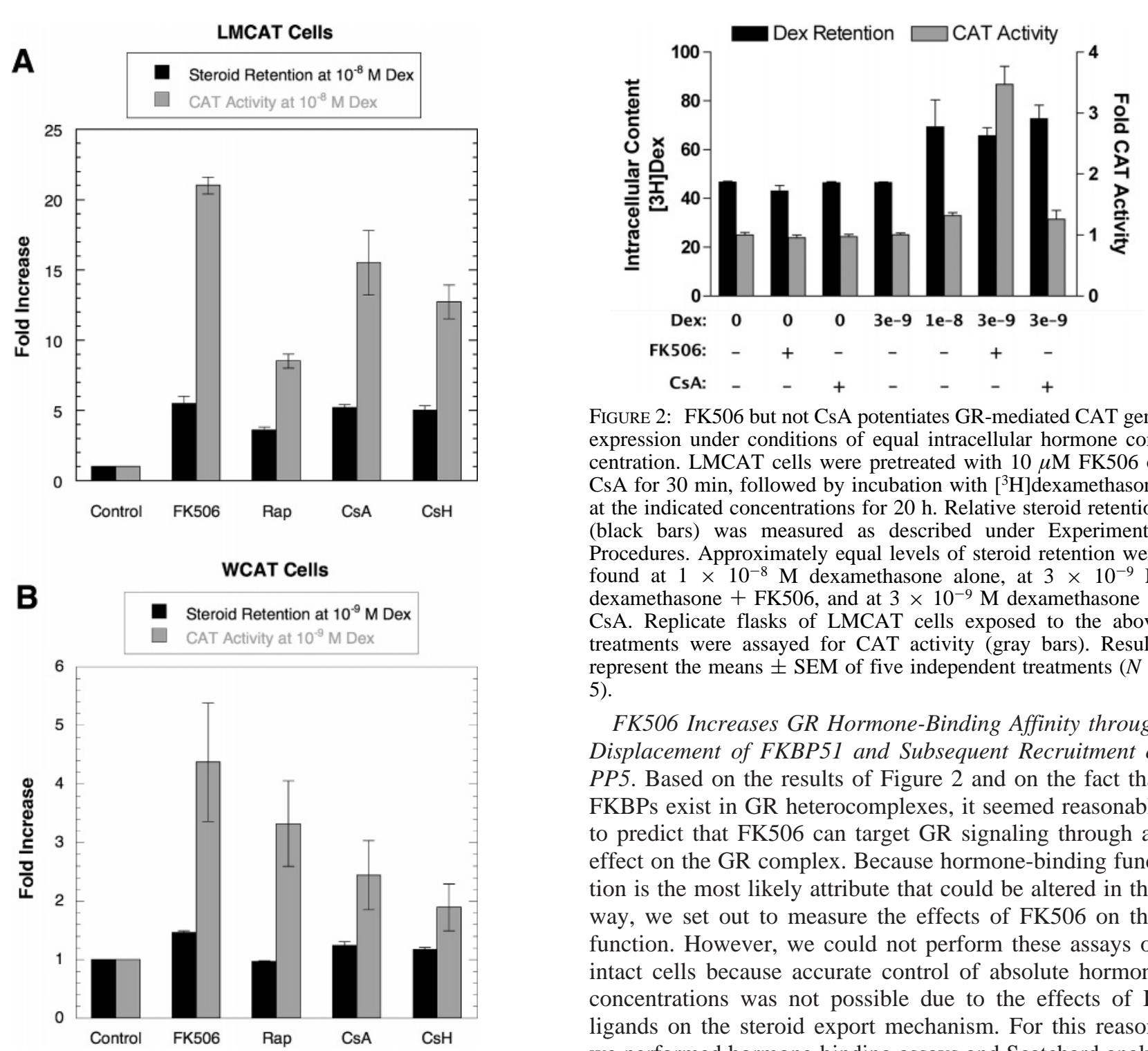

FIGURE 1: Comparison of steroid hormone retention and GRmediated CAT activity in L929 and CHO cells exposed to immunosuppressive ligands. L929 and CHO cells were stably transfected with the pMMTV-CAT reporter to yield LMCAT (A) and WCAT (B) cells, respectively. Each cell line was pretreated with $10 \mu \mathrm{M}$ FK506, rapamycin, CsA, or $\mathrm{CsH}$ for $30 \mathrm{~min}$ prior to hormone addition at the indicated concentrations. Measurements of intracellular hormone concentration and GR-mediated CAT activity were as described under Experimental Procedures. Results represent the means \pm SEM of five independent treatments $(N=$ $5)$.

jected to $1 \times 10^{-8} \mathrm{M}$ hormone alone. We therefore assayed for GR-mediated CAT activity under these conditions (Figure 2 , gray bars). The results show a small increase in CAT activity at $1 \times 10^{-8} \mathrm{M}$ hormone alone but a much larger CAT response at $3 \times 10^{-9} \mathrm{M}$ hormone plus FK506. Thus, FK506 is able to stimulate GR-mediated gene expression, not only by upregulating the amount of hormone in the cell but also by a means that is independent of such a mechanism. Interestingly, CsA had no such effect, suggesting that this ligand may act to potentiate GR solely through the steroid export mechanism. As will be seen below, this difference between FK506 and CsA is likely due to the fact that GR heterocomplexes in LMCAT cells contain both FKBP52 and FKBP51 but no detectable amounts of Cyp40.

FIGURE 2: FK506 but not CsA potentiates GR-mediated CAT gene expression under conditions of equal intracellular hormone concentration. LMCAT cells were pretreated with $10 \mu \mathrm{M}$ FK506 or CsA for $30 \mathrm{~min}$, followed by incubation with $\left[{ }^{3} \mathrm{H}\right]$ dexamethasone at the indicated concentrations for $20 \mathrm{~h}$. Relative steroid retention (black bars) was measured as described under Experimental Procedures. Approximately equal levels of steroid retention were found at $1 \times 10^{-8} \mathrm{M}$ dexamethasone alone, at $3 \times 10^{-9} \mathrm{M}$ dexamethasone + FK506, and at $3 \times 10^{-9} \mathrm{M}$ dexamethasone + CsA. Replicate flasks of LMCAT cells exposed to the above treatments were assayed for CAT activity (gray bars). Results represent the means \pm SEM of five independent treatments $(N=$ $5)$.

FK506 Increases GR Hormone-Binding Affinity through Displacement of FKBP51 and Subsequent Recruitment of PP5. Based on the results of Figure 2 and on the fact that FKBPs exist in GR heterocomplexes, it seemed reasonable to predict that FK506 can target GR signaling through an effect on the GR complex. Because hormone-binding function is the most likely attribute that could be altered in this way, we set out to measure the effects of FK506 on this function. However, we could not perform these assays on intact cells because accurate control of absolute hormone concentrations was not possible due to the effects of IP ligands on the steroid export mechanism. For this reason, we performed hormone-binding assays and Scatchard analysis following FK506 treatment of GR complexes present in cytosolic extracts of LMCAT cells (Figure 3). Additionally, LMCAT cell extracts were exposed to a TPR domain peptide derived from the PP5 sequence (18) which had been shown by Pratt and co-workers to effectively out-compete FKBP52, Cyp40, and Hop for binding to the TPR recognition domain of Hsp90 (26). The results show a large increase in hormonebinding affinity in cytosols exposed to FK506 or TPR peptide ( $K_{\mathrm{d}}=3.4$ and $5.7 \mathrm{nM}$, respectively) compared to cytosols treated with vehicle control $\left(K_{\mathrm{d}}=17.42 \mathrm{nM}\right)$. Curiously, both FK506 and TPR peptide caused a reduction in total hormone-binding sites ( $\beta_{\max }=104.3$ and 126.8 , respectively) as compared to controls $\left(\beta_{\max }=151.8\right)$. To confirm that reduction in binding sites by FK506 was not due to loss of GR protein, we performed quantitative Western blots of GR in the cytosols bound with $100 \mathrm{nM}\left[{ }^{3} \mathrm{H}\right]$ dexamethasone. No difference was observed for GR protein levels in the control and FK506-treated conditions (data not shown).

To test whether the effect of FK506 on GR hormonebinding function was due to alterations in the GR heterocomplex, we co-immunoprecipitated GR with the FiGR antibody against receptor and analyzed the complexes for Hsp90 and TPR protein composition (Figure 4). It can be seen that GR complexes from control cytosols contain Hsp90, 

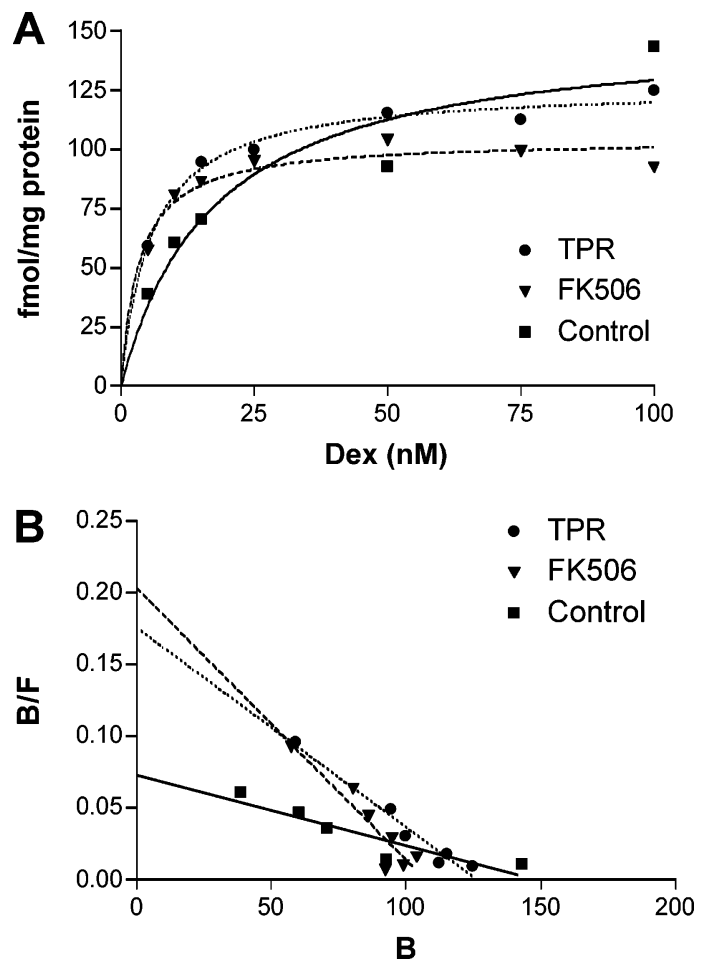

FIGURE 3: FK506 and TPR peptide increase GR hormone-binding affinity. Cytosols derived from LMCAT cells were pretreated with $10 \mu \mathrm{M}$ FK506 or $10 \mu \mathrm{g}$ of TPR peptide for $30 \mathrm{~min}$ prior to addition of $\left[{ }^{3} \mathrm{H}\right]$ dexamethasone. Determination of specific hormone-binding capacities at increasing concentrations of hormone (A) and Scatchard analysis (B) were as described under Experimental Procedures.

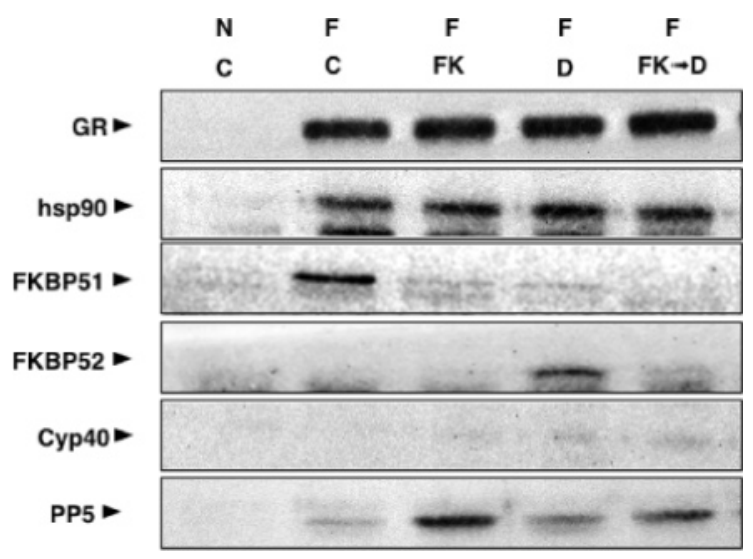

FIGURE 4: FK506 causes release of GR-associated FKBP51 and replacement with PP5, while blocking hormone-induced recruitment of FKBP52. Aliquots of LMCAT cytosol were treated for $1 \mathrm{~h}$ on ice with $10 \mu \mathrm{M}$ FK506 (FK), vehicle control (C), dexamethasone $(\mathrm{D})$, or FK506 prior to dexamethasone $(\mathrm{FK} \rightarrow \mathrm{D})$, followed by an additional incubation for $1 \mathrm{~h}$ at $37^{\circ} \mathrm{C}$. GR complexes were then immunoadsorbed with nonimmune antibody (NI) or FiGR antibody against GR (F) and analyzed for the presence of GR, Hsp90, and TPR proteins by Western blotting, as described under Experimental Procedures. Results are representative of three independent treatments $(N=3)$.

FKBP51, and PP5 but no detectable amount of FKBP52 or Cyp40. In cytosols treated with hormone (dexamethasone), the major response was a loss of FKBP51 from the GR complex and a gain of FKBP52. This hormone-induced swapping of FKBP immunophilins is consistent with our earlier observation in which chilled intact cells were treated with dexamethasone (2). In response to FK506, the major effect seen is loss of FKBP51 and a gain of PP5. In this case, no recruitment of FKBP52 was observed. This result is consistent with the fact that both FKBP51 and FKBP52 bind FK506 and shows that FK506 can disrupt the interaction of each protein with the TPR acceptor site on Hsp90. This result also demonstrates that the TPR acceptor sites on Hsp90 liberated by release of FKBP51 can be occupied by PP5. In cytosols treated with FK506 before dexamethasone, hormoneinduced recruitment of FKBP52 was blocked, and once again an increase in GR-associated PP5 was observed. As above, these effects of FK506 on the GR complex require brief warming of the cytosols to $37^{\circ} \mathrm{C}$.

Taken together, these observations suggest that FK506 can increase GR hormone-binding affinity by causing removal of FKBP51 from GR complexes and replacement with PP5. As discussed below, this effect of FK506 is consistent with the model of Scammell and others (3) in which FKBP51 in GR heterocomplexes is inhibitory with respect to this function.

FKBP52 Is Required for Optimal Response of GR to Hormone. To this point, we have shown that FK506 potentiation of GR transactivity can occur, at least in part, by alterations to the TPR protein composition of GR complexes which, in turn, cause upregulation of GR hormonebinding function. Because FK506 did this by causing a loss of FKBP51 and gain of PP5, while preventing hormoneinduced recruitment of FKBP52, we still did not know the role played by FKBP52 in the GR hormone-binding function. To address this issue, we placed the cDNA for rabbit FKBP52 (kindly provided by Michel Renoir) under the control of a tetracycline-inducible vector, followed by stable transfection into LMCAT cells to yield a new cell line, L52MCAT cells (Figure 5A). In Figure 5B, we show that doxycycline treatment of these cells results in time-dependent expression of rFKBP52, using an antibody that recognizes the rabbit form of FKBP52 but not the endogenous mouse cognate. Strong expression of rFKBP52 was observed at 48 $\mathrm{h}$ of doxycycline treatment. This condition was, therefore, used for subsequent experiments.

Because the hormone-binding event causes recruitment of FKBP52 to the GR complex and because FKBP52 is reported to be stimulatory with respect to GR hormone-binding capacity, we reasoned that upregulation of rFKBP52 should cause an increase in hormone-binding affinity by the GR. As can be seen in Figure 6, this appears to be the case. In this experiment, cytosols were prepared from cells treated with doxycycline or appropriate control conditions. The results once again show an increase in GR ligand-binding affinity for cytosols treated with FK506 $\left(K_{\mathrm{d}}=15.6 \mathrm{nM}\right)$ as compared to controls $\left(K_{\mathrm{d}}=32.6 \mathrm{nM}\right)$. However, in cytosols derived from doxycycline-treated L52MCAT cells, GR hormone-binding affinity was even greater than the FK506 control $\left(K_{\mathrm{d}}=10.1 \mathrm{nM}\right)$. Thus, GR complexes that contain or recruit FKBP52 as the predominant TPR protein appear to have the highest ability to bind and retain hormone. [GR complexes treated with FK506 have PP5 as the dominant TPR protein (see Figure 4).] In Western blot experiments, we have shown that the upregulated rFKBP52 does not enter into the GR complex until GR is bound with hormone (data not shown). Taken as a whole, these results show that ligandinduced recruitment of FKBP52 leads to higher hormonebinding affinity on the part of the receptor and that the levels of FKBP52 in L929 cells may be limiting. 


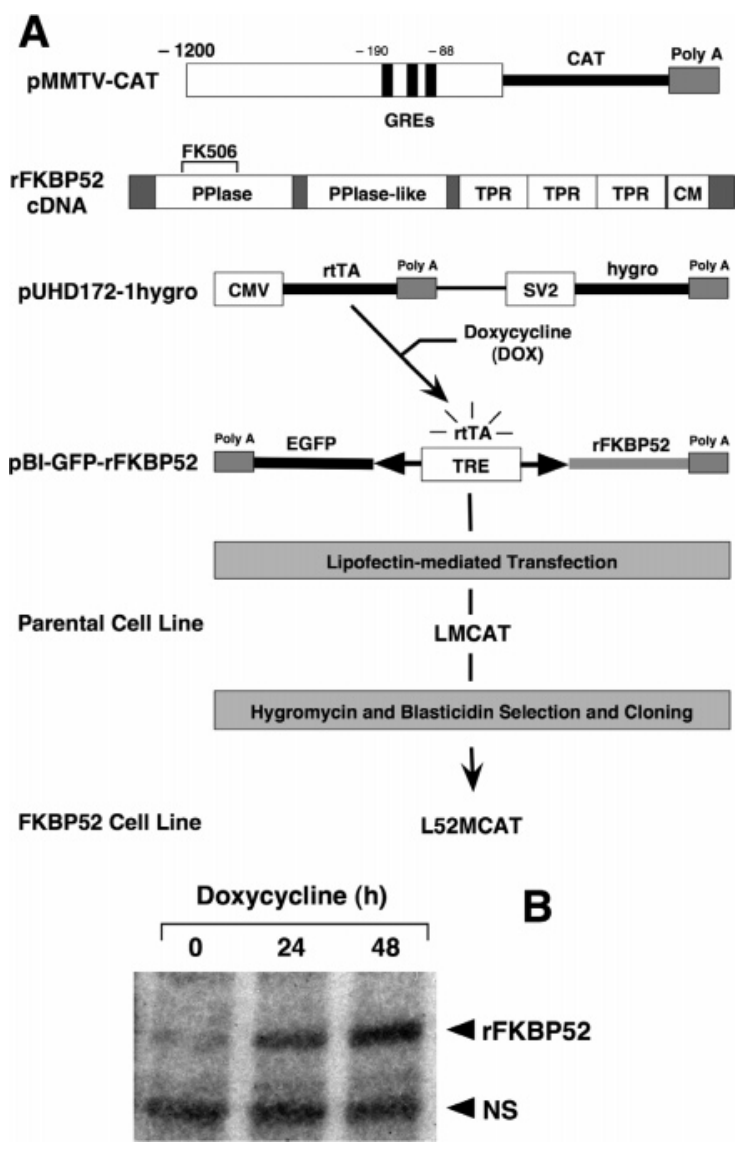

FIGURE 5: Doxycycline-induced upregulation of FKBP52 in LMCAT cells. (A) LMCAT cells containing the pMMTV-CAT reporter were stably transfected with a doxycycline-inducible vector controlling expression of rabbit FKBP52 cDNA to yield L52MCAT cells. (B) Detection of rFKBP52 in L52MCAT cells by Western blotting following 24 and $48 \mathrm{~h}$ treatment with $10 \mu \mathrm{g} / \mathrm{mL}$ doxycycline.

If upregulation of rFKBP52 can increase hormone-binding affinity, then effects on downstream events, such as GR transactivity, should also occur. This was tested by measuring hormone-induced CAT activity in L52MCAT cells treated with or without doxycycline (Figure 7). In the first experimental design (Figure 7A), upregulation of rFKBP52 caused GR-mediated CAT expression to increase at $1 \times 10^{-7} \mathrm{M}$ dexamethasone but not at $1 \times 10^{-8}$ or $1 \times 10^{-6} \mathrm{M}$ hormone. In the second experiment (Figure 7B), the hormone dose dependence was repeated with more increments of hormone between the concentrations of $1 \times 10^{-8}$ and $1 \times 10^{-7} \mathrm{M}$. For comparison, a second condition was added in which cells were treated with FK506 prior to addition of hormone but in the absence of rFKBP52 upregulation (no doxycycline). The results show that upregulation of rFKBP52 can increase GR transactivity at 75 and $100 \mathrm{nM}$ hormone but not at other concentrations. In contrast, treatment with FK506 caused GR transactivity to increase, starting at $10 \mathrm{nM}$ hormone and continuing until $100 \mathrm{nM}$. It is interesting to note that potentiation by FK506 was greater than rFKBP52 upregulation even at $100 \mathrm{nM}$ hormone and that FK506 caused repression of GR transactivity (relative to control and rFKBP52) at $1 \mu \mathrm{M}$ hormone. These results are consistent with a model in which FK506 can affect GR both by increasing intracellular hormone concentration and by causing FKBP51/PP5 interchange, while rFKBP52 upregulation can only affect GR directly at the complex by increasing
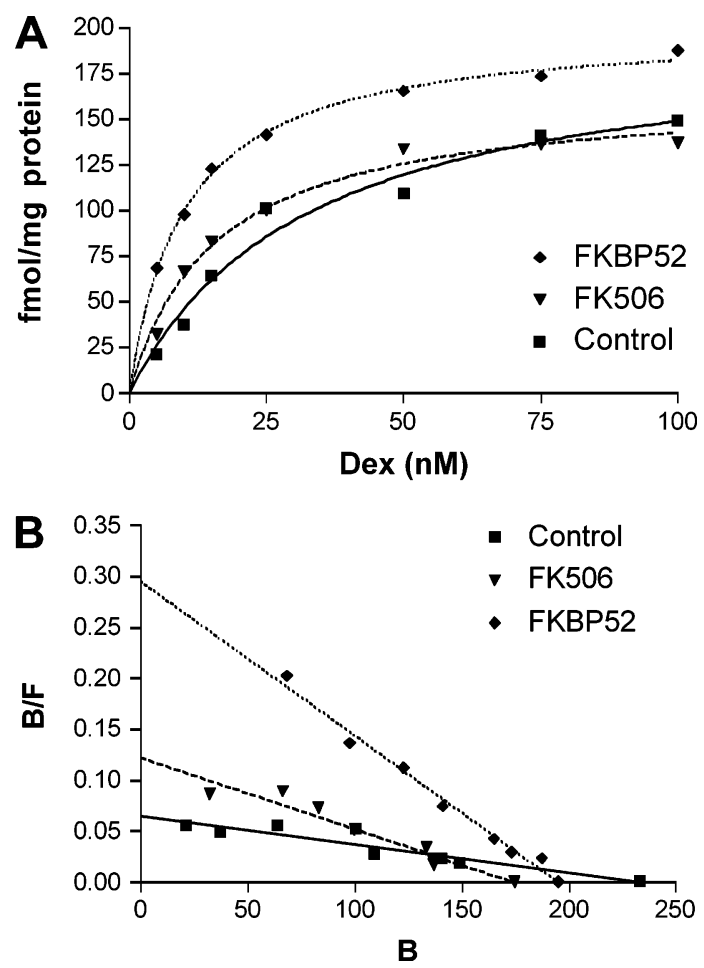

FIGURE 6: Doxycycline-induced upregulation of FKBP52 increases GR hormone-binding affinity to a greater extent than FK506. L52MCAT cells were treated with or without doxycycline for 48 $\mathrm{h}$, followed by preparation of cytosols and analysis of hormonebinding capacity, as described under Experimental Procedures. (A) Specific hormone-binding capacities at increasing concentrations of $\left[{ }^{3} \mathrm{H}\right]$ dexamethasone. (B) Scatchard analysis. Results are representative of three independent treatments $(N=3)$.

the amount of FKBP52 that can interact with receptor once it has bound hormone. Just as important, the results of Figures 6 and 7 show that in mouse L929 cells, at least, FKBP52 may be a limiting factor in GR activity. This would suggest that FKBP52, as compared to FKBP51 and PP5, is required for optimal GR activity in most, if not all, cells.

\section{DISCUSSION}

The importance of immunosuppressive ligands and TPR proteins to glucocorticoid receptor signaling has been known for some time. However, the mechanisms by which these factors control receptor responses are poorly understood, especially in mammalian cells. In this work, we have increased our understanding of these mechanisms by showing that both FK506 and FKBP52 act to control GR responses through alterations in the ability of receptor to bind hormone. FK506 was found to increase GR hormone-binding affinity and decrease total GR-binding sites by causing release of GR-associated FKBP51 and replacement with PP5. The ability of FK506 to potentiate GR activity through a mechanism involving inhibition of steroid exporters was also demonstrated. Thus, with respect to FK506, it is now clear that this ligand can control GR activity on two fronts, as illustrated in the model of Figure 8. According to this model, FK506 can effectively increase the intracellular concentration of steroid by blocking its export from the cells, while increasing the ability of GR to bind hormone through the FKBP51/PP5 interchange. This dual-action model of FK506 can now explain most known responses of GR to this ligand. For example, the ability of FK506 to potentiate receptor 

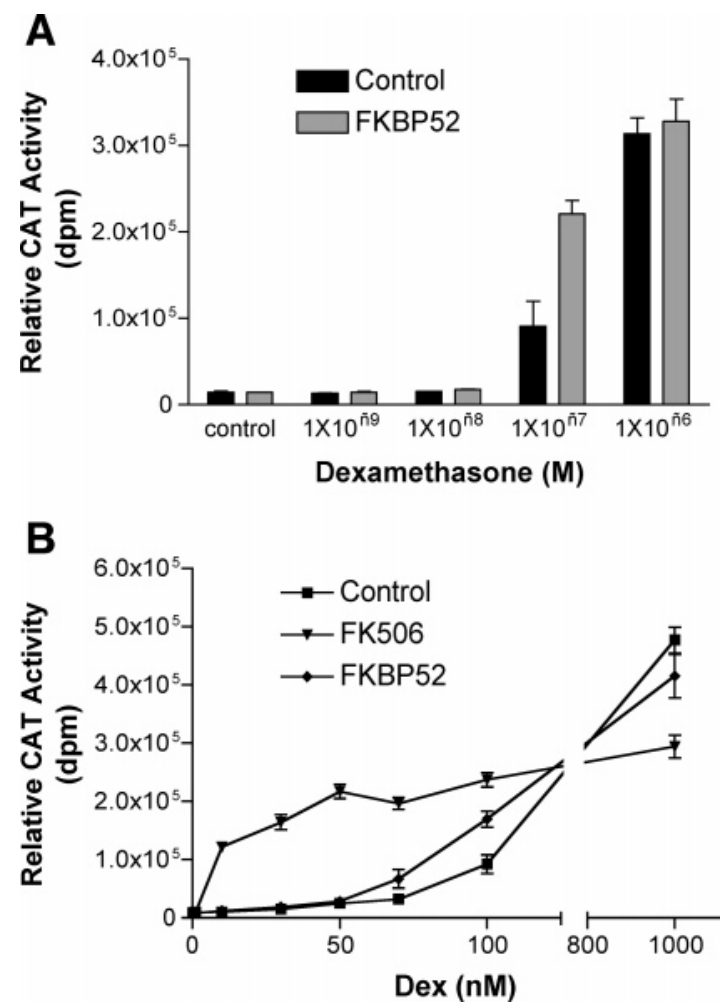

FIGURE 7: Upregulation of FKBP52 in L52MCAT cells increases GR transactivity at intermediate concentrations of hormone. (A) L52MCAT cells were treated with or without doxycycline for 48 $\mathrm{h}$ prior to addition of hormone at the indicated concentrations, followed by assay for CAT activity. Results represent the means \pm SEM of six independent treatments $(N=6)$. (B) Assay for GRmediated CAT expression in L52MCAT cells was repeated using a broader range of hormone concentrations between 10 and 100 $\mathrm{nM}$ dexamethasone. L52MCAT cells were treated with or without doxycycline for $48 \mathrm{~h}$ prior to hormone addition. For comparison, L52MCAT cells (without doxycycline) were also treated with 10 $\mu \mathrm{M}$ FK506 for $1 \mathrm{~h}$ prior to hormone addition. Results represent the means \pm SEM of three independent treatments $(N=3)$.

transactivity at submaximal hormone concentrations is consistent with the ability of FK506 to inhibit steroid export and increase GR hormone-binding affinity. However, inhibition of export could not, by itself, explain the inhibitory effect of FK506 on GR transactivity at high dexamethasone concentrations (this work and refs $8-10$ ). This phenomenon, however, is consistent with the effect of FK506 to cause exchange of FKBP51 for PP5 in the GR complex and a lowering of total hormone-binding sites $\left(\beta_{\max }\right)$. More importantly, we believe our results now provide the basis for understanding the fundamental, unresolved question of why FK506-binding immunophilins exist in GR heterocomplexes. Although endogenous FK506-like ligands have not yet been found, based on our results, it is increasingly likely that the FK506-binding domain of FKBP immunophilins may serve as a regulatory site for control of receptor TPR protein composition.

With respect to Cyp40 and CsA, our data in the L929 cells suggest that CsA potentiates GR transactivity only by increasing intracellular retention, since no effect of CsA is seen at equal intracellular hormone concentration and no effect of CsA on hormone-binding function has been found (data not shown). As illustrated in the model of Figure 8, these properties are consistent with the fact that GR heterocomplexes in L929 cells contain little or no Cyp40. In the
WCAT cells, a moderate potentiation of GR by CsA was observed. This could happen if these cells have at least some CsA-sensitive P-glycoprotein exporter, and we do, in fact, see a small but reproducible increase in hormone retention following CsA treatment. Another potential explanation is that the GR of CHO cells, in contrast to L929 cells, can form a complex with Cyp40. However, using co-IP experiments, we have not been able to detect GR-associated Cyp40 in these cells (data not shown).

Because FK506 can potentiate the GR, in part, by swapping FKBP51 for PP5 while blocking hormone-induced recruitment of FKBP52, it could be argued that FKBP52 may be inhibitory with respect to GR transactivity. We now know that this is not the case, since upregulation of FKBP52 in L929 cells causes both GR hormone-binding affinity and transactivity to increase. Moreover, the increase seen in GR hormone-binding function is greater than that obtained in response to FK506. These results are in agreement with a recent report from the Smith laboratory showing that mammalian FKBP52 expressed in yeast will increase rat GR hormone-binding affinity (5). On the basis of our work, this ability of FKBP52 now appears to hold true for GR of mammalian cells.

We can now propose a model for the contributions of FKBP52, FKBP51, and PP5 to GR functionality (Figure 9). According to this model, the basal state of inactive GR heterocomplexes is one in which FKBP51 is the predominant TPR protein. In this state, the GR exhibits the lowest intrinsic hormone-binding capacity and, in the absence of contributions from FKBP52 or PP5, exhibits the lowest level of GR transactivity. As illustrated in the model, when GR acquires PP5, it now has intermediate hormone-binding and transcriptional activities. So far, however, this state of GR has only been achieved by "artificial" means, namely, through treatment with FK506. It remains to be seen whether and how the PP5 state of receptor are generated in the normal course of GR signaling events. When the GR acquires FKBP52, it now exhibits the highest levels of hormonebinding and transcriptional activities. It is reasonable to assume that the increased transactivity seen is completely due to upregulation of the hormone-binding function. However, because FKBP52 recruitment (along with dynein) appears to be involved in translocation of the GR into the nucleus $(2,27)$, it is possible that FKBP52 may also increase transactivity by increasing the efficiency of GR targeting to the nucleus or even to promoter sites. The mechanism by which FKBP52 increases GR hormone-binding affinity is still far from clear. One possibility is that FKBP52 simply stabilizes or alters the GR/Hsp90 interaction, which is known to occur in the hormone-binding domain (28). But it has been shown by the Pratt and Smith laboratories $(29,30)$ that FKBP52 can also contact the GR at the hormone-binding domain, which FKBP51 may or may not be able to do.

Because upregulation of FKBP52 in L929 cells caused the hormone response to increase, it can be argued that FKBP52 levels in these cells are limiting. This would suggest that the highly variable responses seen to steroids across cell types may be due, in large measure, to the relative concentration levels of the TPR proteins expressed. Thus, cells with high levels of FKBP51 may be weak responders to hormone, while those with high levels of FKBP52 may be strong responders. Although less clear, in cells with high levels of 


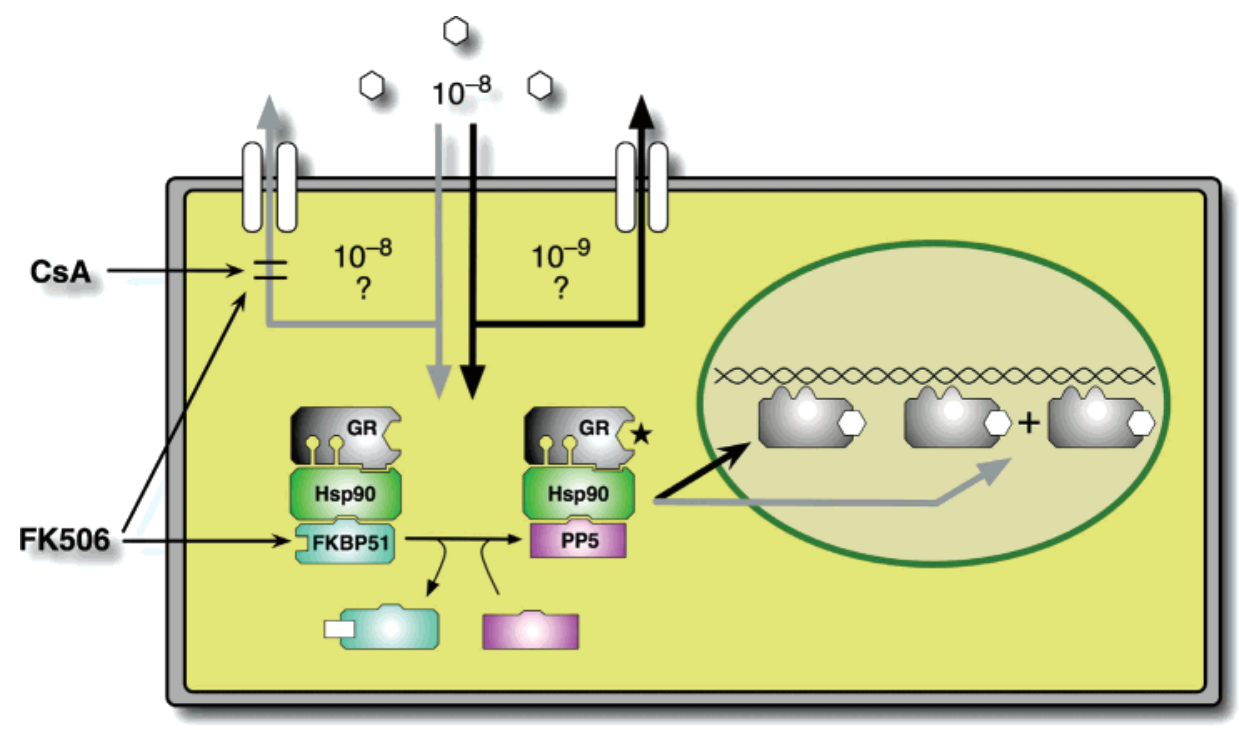

FIGURE 8: Model for the dual action of FK506 on the GR response to hormone. We have shown that the hormone-free GR complex of LMCAT cells (mouse L929 cell parentage) contains FKBP51 as the predominant TPR protein. FK506 causes FKBP51 to be released from the complex, while simultaneously preventing hormone-induced recruitment of FKBP52 to the complex. The free TPR-acceptor domain of Hsp90 thus generated is occupied by PP5, leading to a state of GR that has increased hormone-binding affinity with reduced total binding sites $(*)$. In addition to this mode of potentiation, FK506 also causes the intracellular concentration of hormone to increase, presumably by blocking membrane-based steroid exporters. In contrast, cyclosporin A (CsA) only affects GR in these cells through inhibition of the steroid export mechanism. The inability of CsA to alter GR hormone-binding function is reflected in the fact that the GR complexes of L929 cells contain little, if any, Cyp40.

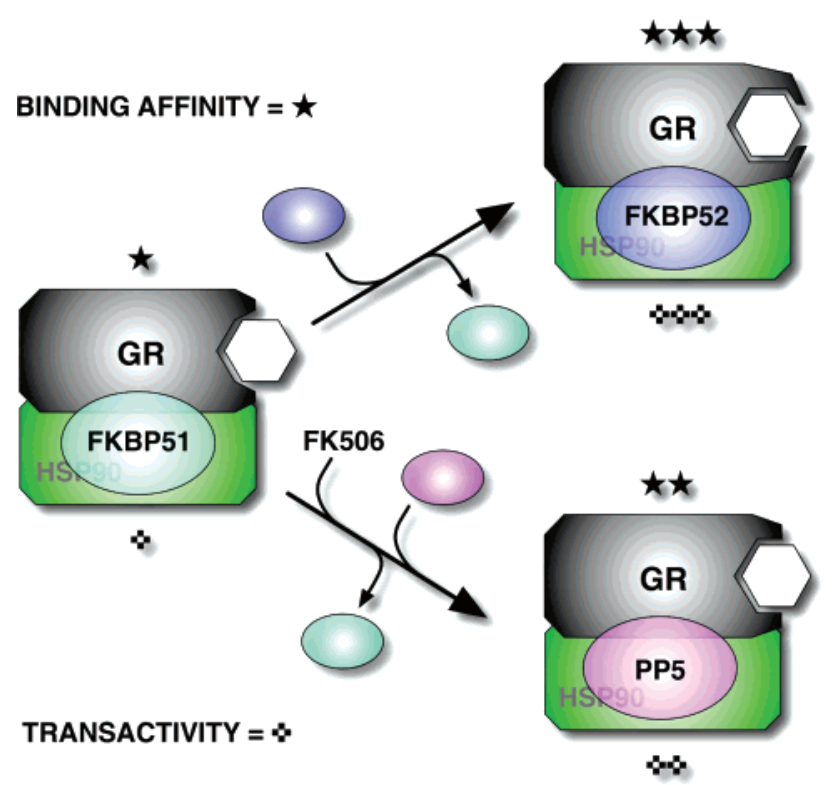

FIGURE 9: Model for the roles of FK506 and TPR proteins on GR hormone-binding and transactivation functions. We have shown that FK506 can increase the hormone-binding affinity of GR through a mechanism that involves displacement of FKBP51 and replacement with PP5. In the absence of FK506, binding of hormone to the GR results in replacement of FKBP51 with FKBP52. Because upregulation of FKBP52 caused GR hormone-binding function to increase to a level greater than that seen when GR is occupied by PP5 (FK506 treatment), we can now postulate the following hierarchy for the contribution of TPR proteins to GR hormone-binding capacity: FKBP51 < PP5 < FKBP52. As reflected in each complex, it is likely that the TPR proteins cause intrinsic changes in the conformation of the GR ligand-binding domain, either through allosteric effects on Hsp90 or through direct contact. In all states shown, downstream transactivity functions correlate with receptor hormone-binding affinity.

PP5, an intermediate response to hormone may be seen. How cells with high levels of Cyp40 would respond is even more uncertain. In our L929 cells, a strong signal for Cyp40 is seen in cytosolic extracts (data not shown), yet very little, if any, Cyp40 appears to enter into the GR complex in either the presence or absence of hormone. This suggests that, in addition to levels of expression, specific receptors may preferentially enter into complexes with only certain TPR proteins. This speculation is consistent with the fact that studies showing Cyp40 in receptor complexes have mostly been for the estrogen receptor $(31,32)$. Such a mechanism may also explain the results obtained by Toft and co-workers in which CsA was found to have opposite effects on progesterone receptor action in human T47D versus mouse L929 cells (33). Lastly, preferential association of receptors with TPR proteins may also form the basis for controlling the receptor subcellular localization in the absence of hormone, a property of receptors known to vary widely across receptor class and tissue type.

\section{ACKNOWLEDGMENT}

The authors thank Drs. Jack Bodwell, Karen Leach, William Pratt, and Michel Renoir for the kind gifts of FiGR antibody, UPJ56 antibody, TPR peptide, and rabbit FKBP52 cDNA, respectively.

\section{REFERENCES}

1. Pratt, W. B., and Toft, D. O. (1997) Steroid receptor interactions with heat shock protein and immunophilin chaperones, Endocr. Rev. 18, 306-360.

2. Davies, T. H., Ning, Y. M., and Sanchez, E. R. (2002) A new first step in activation of steroid receptors: hormone-induced switching of FKBP51 and FKBP52 immunophilins, J. Biol. Chem. 277, 4597-4600.

3. Reynolds, P. D., Ruan, Y., Smith, D. F., and Scammell, J. G. (1999) Glucocorticoid resistance in the squirrel monkey is associated with overexpression of the immunophilin FKBP51, $J$. Clin. Endocrinol. Metab. 84, 663-669.

4. Denny, W. B., Valentine, D. L., Reynolds, P. D., Smith, D. F., and Scammell, J. G. (2000) Squirrel monkey immunophilin 
FKBP51 is a potent inhibitor of glucocorticoid receptor binding, Endocrinology 141, 4107-4113.

5. Cheung-Flynn, J., Roberts, P. J., Riggs, D. L., and Smith, D. F. (2003) C-terminal sequences outside the tetratricopeptide repeat domain of FKBP51 and FKBP52 cause differential binding to Hsp90, J. Biol. Chem. 278, 17388-17394.

6. Ivery, M. T. (2000) Immunophilins: switched on protein binding domains, Med. Res. Rev. 20, 452-484.

7. Fretz, H., Albers, M. W., Galat, A., Standaert, R. F., Lane, W. S., Burakoff, S. J., Bierer, B. E., and Schreiber, S. L. (1991) Rapamycin and FK506 binding proteins (immunophilins), J. Am. Chem. Soc. 113, 1409-1411.

8. Ning, Y. M., and Sanchez, E. R. (1993) Potentiation of glucocorticoid receptor-mediated gene expression by the immunophilin ligands FK506 and rapamycin, J. Biol. Chem. 268, 6073-6076.

9. Le Bihan, S., Marsaud, V., Mercier-Bodard, C., Baulieu, E. E., Mader, S., White, J. H., and Renoir, J. M. (1998) Calcium/ calmodulin kinase inhibitors and immunosuppressant macrolides rapamycin and FK506 inhibit progestin- and glucocorticosteroid receptor-mediated transcription in human breast cancer T47D cells, Mol. Endocrinol. 12, 986-1001.

10. Deppe, C. E., Heering, P. J., Viengchareun, S., Grabensee, B., Farman, N., and Lombes, M. (2002) Cyclosporine A and FK506 inhibit transcriptional activity of the human mineralocorticoid receptor: a cell-based model to investigate partial aldosterone resistance in kidney transplantation, Endocrinology 143, 19321941.

11. Shimoishi, K., Kai, H., Kabu, K., Komori, T., Maruyama, T., and Otagiri, M. (2001) FK506 (tacrolimus) increases rat alpha1-acid glycoprotein expression in liver and primary cultured hepatocytes, Eur. J. Pharmacol. 420, 91-95.

12. Migita, K., Tanaka, H., Okamoto, K., Yoshikawa, N., Ichinose, Y., Urayama, S., Yamasaki, S., Ida, H., Kawabe, Y., Kawakami, A., and Eguchi, K. (2000) FK506 augments glucocorticoidmediated cyclooxygenase-2 down-regulation in human rheumatoid synovial fibroblasts, Lab. Invest. 80, 135-141.

13. Kralli, A., and Yamamoto, K. R. (1996) An FK506-sensitive transporter selectively decreases intracellular levels and potency of steroid hormones, J. Biol. Chem. 271, 17152-17156.

14. Chalepakis, G., Arnemann, J., Slater, E., Bruller, H.-J., Gross, B., and Beato, M. (1988) Differential gene activation by glucocorticoids and progestins through the hormone regulatory element of mouse mammary tumor virus, Cell 53, 371-382.

15. Sanchez, E. R., Hirst, M., Scherrer, L. C., Tang, H. Y., Welsh, M. J., Harmon, J. M., Simons, S. S. J., Ringold, G. M., and Pratt, W. B. (1990) Hormone-free mouse glucocorticoid receptors overexpressed in Chinese hamster ovary cells are localized to the nucleus and are associated with both hsp70 and hsp90, J. Biol. Chem. 265, 20123-20130.

16. Hirst, M. A., Northrop, J. P., Danielsen, M., and Ringold, G. M. (1990) High level expression of wild-type and variant mouse glucocorticoid receptors in Chinese hamster ovary cells, Mol. Endocrinol. 4, 162-170.

17. Nordeen, S. K., Green, P. P. I. I. I., and Fowlkes, D. M. (1987) A rapid, sensitive, and inexpensive assay for chloramphenicol acetyltransferase, DNA 6, 173-178.

18. Chen, M. S., Silverstein, A. M., Pratt, W. B., and Chinkers, M. (1996) The tetratricopeptide repeat domain of protein phosphatase 5 mediates binding to glucocorticoid receptor heterocomplexes and acts as a dominant negative mutant, J. Biol. Chem. 271, $32315-32320$.

19. Bodwell, J. E., Orti, E., Coull, J. M., Pappin, D. J., Smith, L. I., and Swift, F. (1991) Identification of phosphorylated sites in the mouse glucocorticoid receptor, J. Biol. Chem. 266, 7549-7555.
20. Laemmli, U. K. (1970) Cleavage of structural proteins during the assembly of bacteriophage T4, Nature 227, 680-685.

21. Sanchez, E. R., Meshinchi, S., Tienrungroj, W., Schlesinger, M. J., Toft, D. O., and Pratt, W. B. (1987) Relationship of the 90$\mathrm{kDa}$ murine heat shock protein to the untransformed and transformed states of the L cell glucocorticoid receptor, J. Biol. Chem. 262, 6986-6991.

22. Wadekar, S. A., Li, D., Periyasamy, S., and Sanchez, E. R. (2001) Inhibition of heat shock transcription factor by glucocorticoid receptor, Mol. Endocrinol. 15, 1396-410.

23. Ruff, V. A., Yem, A. W., Munns, P. L., Adams, L. D., Reardon, I. M., Deibel, M. R., Jr., and Leach, K. L. (1992) Tissue distribution and cellular localization of hsp56, an FK506-binding protein. Characterization using a highly specific polyclonal antibody, J. Biol. Chem. 267, 21285-21288.

24. Renoir, J. M., Mercier-Bodard, C., Hoffmann, K., Le, B. S., Ning, Y. M., Sanchez, E. R., Handschumacher, R. E., and Baulieu, E. E. (1995) Cyclosporin A potentiates the dexamethasone-induced mouse mammary tumor virus-chloramphenicol acetyltransferase activity in LMCAT cells: a possible role for different heat shock protein-binding immunophilins in glucocorticosteroid receptormediated gene expression, Proc. Natl. Acad. Sci. U.S.A. 92, 49774981.

25. Boerner, S. A., Tourne, M. E., Kaufmann, S. H., and Bible, K. C. (2001) Effect of P-glycoprotein on flavopiridol sensitivity, Br. J. Cancer 84, 1391-1396.

26. Silverstein, A. M., Galigniana, M. D., Chen, M. S., Owens-Grillo, J. K., Chinkers, M., and Pratt, W. B. (1997) Protein phosphatase 5 is a major component of glucocorticoid receptor ${ }^{\mathrm{hsp}} 90 \mathrm{com}-$ plexes with properties of an FK506-binding immunophilin, J. Biol. Chem. 272, 16224-16230.

27. Galigniana, M. D., Radanyi, C., Renoir, J. M., Housley, P. R., and Pratt, W. B. (2001) Evidence that the peptidylprolyl isomerase domain of the hsp90-binding immunophilin FKBP52 is involved in both dynein interaction and glucocorticoid receptor movement to the nucleus, J. Biol. Chem. 276, 14884-1489.

28. Ning, Y. M., and Sanchez, E. R. (1995) Stabilization in vitro of the untransformed glucocorticoid receptor complex of S49 lymphocytes by the immunophilin ligand FK506, J. Steroid Biochem. Mol. Biol. 52, 187-194.

29. Silverstein, A. M., Galigniana, M. D., Kanelakis, K. C., Radanyi, C., Renoir, J. M., and Pratt, W. B. (1999) Different regions of the immunophilin FKBP52 determine its association with the glucocorticoid receptor, hsp90, and cytoplasmic dynein, J. Biol. Chem. 274, 36980-36986.

30. Riggs, D. L., Roberts, P. J., Chirillo, S. C., Cheung-Flynn, J., Prapapanich, V., Ratajczak, T., Gaber, R., Picard, D., and Smith, D. F. (2003) The Hsp90-binding peptidylprolyl isomerase FKBP52 potentiates glucocorticoid signaling in vivo, EMBO J. 22, 11581167.

31. Ratajczak, T., Hlaing, J., Brockway, M. J., and Hahnel, R. (1990) Isolation of untransformed bovine estrogen receptor without molybdate stabilization, J. Steroid Biochem. 35, 543-553.

32. Ratajczak, T., and Carrello, A. (1996) Cyclophilin 40 (CyP-40), mapping of its hsp90 binding domain and evidence that FKBP52 competes with CyP-40 for hsp90 binding, J. Biol. Chem. 271, 2961-2965.

33. Milad, M., Sullivan, W., Diehl, E., Altmann, M., Nordeen, S., Edwards, D. P., and Toft, D. O. (1995) Interaction of the progesterone receptor with binding protein for FK506 and cyclosporin A, Mol. Endocrinol. 9, 838-847.

BI048503V 